\title{
Asynchronous Clustering of Multihop Wireless Sensor Networks
}

\author{
Serdar Vural, Pirabakaran Navaratnam, Ning Wang, and Rahim Tafazolli \\ Centre for Communication Systems Research, University of Surrey, Guildford, Surrey, GU2 7XH, UK \\ e-mail: \{s.vural, p.navaratnam, n.wang, r.tafazolli\}@surrey.ac.uk
}

\begin{abstract}
Node clustering has been widely studied in recent years for Wireless Sensor Networks (WSN) as a technique to form a hierarchical structure and prolong network lifetime by reducing the number of packet transmissions. Cluster Heads (CH) are elected in a distributed way among sensors, but are often highly overloaded, and therefore re-clustering operations should be performed to share the resource intensive $\mathrm{CH}-$ role. Existing protocols involve periodic network-wide re-clustering operations that are simultaneously performed, which requires global time synchronisation. To address this issue, some recent studies have proposed asynchronous node clustering for networks with direct links from CHs to the data sink. However, for large-scale WSNs, multihop packet delivery to the sink is required since longrange transmissions are costly for sensor nodes. In this paper, we present an asynchronous node clustering protocol designed for multihop WSNs, considering dynamic conditions such as residual node energy levels and unbalanced data traffic loads caused by packet forwarding. Simulation results demonstrate that it is possible to achieve similar levels of lifetime extension by reclustering a multihop WSN via independently made decisions at $\mathrm{CHs}$, without a need for time synchronisation required by existing synchronous protocols.
\end{abstract}

\section{INTRODUCTION}

Wireless Sensor Networks (WSN) are formed of simple sensing devices with limited battery energy that autonomously operate without being recharged for extended periods of time. Some typical applications are environmental or habitat monitoring and military surveillance, requiring the collection of sensory data to a control centre from a large deployment area.

Energy savings are possible in WSNs via exploiting the similarities in the collected data streams generated by sensors that are geographically close. The correlation of harvested data from sensors in the same locality leads to possibilities to summarise the gathered information from multiple sensors in a certain location by producing a single representative data stream. By placing nodes with higher computational power and higher energy stocks at regular locations in the network, a hierarchical structure can be formed, in which such nodes can perform data summarization and reduce the amount of data transferred to the network's data sink. However, WSNs must often be deployed without any infrastructure support and operate in a self-organised manner. Therefore, these data summarization nodes are instead selected from the deployed sensors, which then collect the data traffic in their vicinity. Without human intervention, forming such clusters of sensors is to be performed by the network itself: an autonomous construction of network hierarchy [1]. Each sensor that collects data from a cluster of sensors is called a cluster head $(\mathrm{CH})$.

Although the hierarchical structure obtained by node clustering provides energy savings by means of data summarization, $\mathrm{CH}$ nodes are responsible for processing the collected data streams from sensors and then relaying the summarised information to a next hop $\mathrm{CH}$, to be delivered to the data sink. Therefore, $\mathrm{CH}$ nodes have higher energy consumption rates than ordinary sensors, effectively making them prone to early energy depletion. To prevent this, the $\mathrm{CH}$-role is rotated among network nodes by renewing the set of $\mathrm{CHs}$ at regular intervals, which is a process called re-clustering.

Many node clustering protocols have been proposed as a means to extend network lifetime in WSN data collection scenarios [1]. These protocols divide the runtime into time rounds. Each round starts with a short re-clustering phase followed by a considerably longer data transmission phase in order to obtain a high fraction of time devoted to data delivery rather than topology management. However, protocols based on time-rounds require network-wide time synchronisation, so that nodes can simultaneously engage in this short reclustering phase and perform cooperative operations to elect the new set of CHs. Some recent studies [2] [3] [4] propose asynchronous clustering to avoid this requirement, however the proposed protocols assume direct links from $\mathrm{CHs}$ to the data sink, and therefore are limited to scenarios in which sensors can reach the sink in a single hop.

In this paper, we present the Asynchronous Clustering Protocol (ACP), designed for multihop WSN data collection scenarios. Sensor nodes dynamically change their transmission ranges to effectively modify the network hierarchy, considering residual node energy levels as well as the unbalanced traffic loads on $\mathrm{CHs}$ caused by multihop packet forwarding. In contrast to existing clustering protocols proposed for multihop WSNs, ACP does not require network-wide timesynchronisation [2] [5] [6] [7], estimation of inter-node distances [6], specific network topologies [7], or static estimations of energy consumption levels over multihop paths [7]. ACP is compared with the Unequal Cluster-based Routing (UCR) protocol in multihop WSNs in terms of gains in average throughput at the data sink and network lifetime. Simulation results demonstrate that ACP can achieve similar levels of network lifetime extension, while achieving higher throughput and lower node outage. 
The paper is organised as follows. First, Sec. II provides a brief review of node clustering protocols in WSNs. Then, Sec. III describes ACP in detail. Performance results are provided in Sec. IV. Finally, Sec. V concludes the paper.

\section{RELATED WORK}

Node clustering in WSNs has been extensively studied in the past decade [1], with a number of protocols proposed to save limited sensor energy so as to extend network lifetime. The first generation of clustering protocols are designed for networks with direct links between $\mathrm{CHs}$ and the network's data sink [8] [9] [10]. The LEACH protocol [8] provides the first example of how a WSN can be autonomously organised to extend its lifetime. To achieve this, the strategy is to equalise the time fraction that different nodes take the load-intensive $\mathrm{CH}$ role. Starting with equal energy levels, each node picks a probability value to decide whether to act as a $\mathrm{CH}$, based on the number of times it has recently claimed the $\mathrm{CH}$ role. An improved version of $\mathrm{LEACH}$, called M-LEACH, is proposed in [11], in which nodes in a cluster deliver data to the $\mathrm{CH}$ over multihop paths and can thus achieve energy savings. Similarly, k-hop forwarding from cluster members towards the $\mathrm{CH}$ is included in [9] with simpler sensors that have a fixed radius rather than an adjustable one.

Instead of the received signal strength (RSS) from neighbour $\mathrm{CHs}$, later clustering protocols introduce different metrics for sensors when making a decision on which $\mathrm{CH}$ to join. EECS [12] proposes a weighted cost function that takes into account sensor-to- $\mathrm{CH}$ and $\mathrm{CH}$-to-sink distances to consider the transmission costs. HEED [5] introduces average reachability power, which is the average minimum transmission power to reach a $\mathrm{CH}$ from its neighbour nodes. This is used as an estimate of the prospective intra-cluster communication costs if the node chooses to act as a $\mathrm{CH}$.

Residual node energy levels have been considered in clustering protocols to assign the $\mathrm{CH}$ role to resource-rich sensors. EEHC [13] is one example, which is designed for networks with direct links to the data sink from CHs. Similarly, HEED [5] takes into account residual node energy levels and uses $\mathrm{CH}$ node degrees to uniformly distribute the $\mathrm{CHs}$ in the network and provide high connectivity. Similar to HEED, DEEC [14] can provide fairer $\mathrm{CH}$ election in terms of energy levels, but all nodes need to know the most recent average node energy in the network. An improvement of HEED is the VCA protocol [15] which uses a voting mechanism to elect the $\mathrm{CHs}$ and regular neighbourhood messages to inform neighbour nodes of residual energy levels.

Apart from sensors' residual battery energy, a high traffic load is another factor that leads to early energy depletion. Towards this, some approaches [6] [7] [16] have considered the imbalance in traffic load between different sensors, and as a remedy, clusters of different sizes are formed, i.e. the $\mathrm{CH}$ communication range with cluster members. UCS [16] considers powerful nodes pre-deployed in the network to serve as CHs, whereas others, e.g. UCR [6] and EC [7], elect $\mathrm{CHs}$ in regular time rounds. These protocols are aimed at improving network lifetime via elimination of network hot-spots caused by the imbalance in traffic loads on $\mathrm{CHs}$ due to multihop forwarding to the data sink. UCR needs to estimate inter-node distances using RSS levels to adjust node transmission ranges. In comparison, EC can provide longer network lifetime; however it relies on estimation of end-to-end packet delivery energy costs in a specific rectangular topology that is divided into virtual regions.

The common mechanism in node clustering in WSNs is that network runtime is divided into time rounds which start with a short re-clustering stage followed by a longer data transmission stage. In order to rotate the load-intensive $\mathrm{CH}$ role, nodes periodically perform re-clustering operations at each re-clustering stage to elect a new set of $\mathrm{CHs}$ that will serve the network during the next round. Recently, some studies have emphasized the need for a fully asynchronous operation in a self-organized WSN, which does not require time rounds [2] [3] [4]. These asynchronous node clustering protocols are designed for networks with single hops from CHs to the data sink and are not applicable to multihop WSNs, since in such networks it is essential to consider the multihop traffic loads. In contrast, this paper presents an asynchronous protocol specifically designed for multihop WSNs.

\section{Asychronous Clustering Protocol (ACP)}

$\mathrm{ACP}$ is a node clustering protocol, in which $\mathrm{CH}$ nodes make independent decisions to perform re-clustering operations. In contrast to conventional clustering protocols, ACP is not based on time-rounds and synchronised periodic reclustering operations. When certain criteria are met, $\mathrm{CH}$ nodes initiate such operations either within their own clusters or over multiple neighbour clusters, covering a section of the network. Similar to most existing clustering protocols [5] [6] [7], each node $i$ keeps a probability value $P(i)$, called the $\mathrm{CH}$-probability, which reflects its likelihood to claim the $\mathrm{CH}$ role. ACP updates $\mathrm{CH}$-probability values dynamically, based on three main node variables: (i) residual node energy level $E(i)$, (ii) latest incoming data traffic rate $r_{i n}(i)$, and (iii) hop distance $h(i)$ to the sink. Cluster sizes are unequal, which is a strategy to equalise energy consumption rates at nodes.

\section{A. Network Model}

ACP is designed for data collection scenarios in largescale multihop WSNs, which deliver collected sensor data to a network sink situated at a random location in the network area. Each sensor node is attached to a single $\mathrm{CH}$, which collects the data generated by the sensors in its cluster, processes the data streams and summarises them into a single data stream, and then forwards the data packets to a next hop $\mathrm{CH}$ towards the data sink. The cluster size of a $\mathrm{CH}$ is its transmission range when communicating with cluster members. Cluster sizes are dynamically modified by re-clustering operations, which changes the network hierarchy over time. Data and control packet transmissions between $\mathrm{CHs}$ have a range of $R$, which is computed as outlined in [17]. 


\section{B. Re-clustering in $A C P$}

ACP has two re-clustering operations ${ }^{1}$, ICR and MCR, that enable dynamic change of the $\mathrm{CH}$-roles, as explained next.

1) Intra-Cluster Re-clustering (ICR): ICR is based on the idea that a $\mathrm{CH}$ node may prefer to periodically compare its residual energy with those of the nodes in its cluster, and transfer its role to the most suitable one. This is performed in an attempt to balance the $\mathrm{CH}$ role, while limiting the transfer operations within individual cluster boundaries. Denoting the set of cluster members of a $\mathrm{CH} j$ by $C(j)$, an ICR check $^{2}$ is:

Find a member $k \mid E(k)>\theta_{I N} * E(j), \quad k \in C(j)$,

where $\theta_{I N}$ is a threshold coefficient, and $E($.$) denotes node$ energy. An ICR operation is performed only if the ICR check holds for a member $k$ with the highest residual energy. However, ACP allows a $\mathrm{CH}$ to transfer its role to a member node only if that node currently has connectivity to another $\mathrm{CH}$. To ensure this, the following procedure is followed. $\mathrm{CH}$ $j$ considers node $k$ as a candidate and checks if $k$ has any $\mathrm{CH}$ neighbours other than $\mathrm{CH} j$, by sending a BEACON packet to node $k$. Upon reception of the BEACON from $\mathrm{CH} j$, if node $k$ does not have another $\mathrm{CH}$ neighbour ${ }^{3}$, then it replies with a BEACON to $\mathrm{CH} j$, which then cancels the ICR and does not transfer its role. Otherwise, node $k$ transmits a BEACON to its best $\mathrm{CH}$ neighbour bch (highest RSS) to check whether it is still available as a $\mathrm{CH}$. After later BEACON transmissions from $\mathrm{CH}$ bch to node $k$ and then to $\mathrm{CH} j$, the $\mathrm{CH}$ role can be transferred only if $\mathrm{CH} b c h$ is available. This is a policy towards keeping the network connected. Alternative nodes in the cluster are not tried consecutively, in order to keep the ICR as short as possible. This minimizes the data transfer interruption which can be caused by a long series of BEACON transmissions, especially for clusters with many members.

After a confirmation is received from its prospective $\mathrm{CH}$, $\mathrm{CH} j$ first announces to its $\mathrm{CH}$ neighbours that it is about to transfer its role, with the transmission of a CHCANCEL packet. Neighbour CHs that have picked $j$ as their next hop now check the availability of an alternative next hop $\mathrm{CH}$ via a $\mathrm{BEACON}$ packet, by going through the list of $\mathrm{CHs}$ in a descending order of RSS. CH $j$ also transmits a CHCHANGE to its members, which includes the new CH's ID $k$ and the latest baseline probability $p_{h(j)}$ (see Sec. III-C3) computed by $\mathrm{CH} j$. Upon receiving the CHCHANGE, the new $\mathrm{CH} k$ first updates its $\mathrm{CH}$ probability $P(k)$ by Eqn. 5 using the baseline probability $p_{h(j)}$ received from the former $\mathrm{CH} j$. Other member nodes also update their local copies of the baseline probability. Then, $\mathrm{CH}$ $k$ transmits an ANNOUNCE packet with a range of $r(k)$ (see Sec. III-C1); receiving nodes not linked to a $\mathrm{CH}$ join $\mathrm{CH} k$. Member nodes outside $\mathrm{CH} k$ 's range join a nearby $\mathrm{CH}$ with the highest RSS; or if no alternative $\mathrm{CH}$ exists, they start a reclustering timer (Sec. III-B2) to become a prospective $\mathrm{CH}$.

\footnotetext{
${ }^{1} \mathrm{~A} \mathrm{CH}$ and its cluster can participate in either ICR or MCR at a time.

${ }^{2}$ ICR checks are limited to individual cluster boundaries, which avoids excessive control packet exchange over the whole network.

${ }^{3}$ The neighbour $\mathrm{CH}$ with the highest RSS is discovered via CHSIGNALs, see Sec. III-D3.
}

2) Multi-Cluster Re-clustering (MCR): $\mathrm{MCR}$ refreshes the cluster structure over a wider area than a single cluster's coverage area. The operation is aimed at reducing the energy imbalance across multiple clusters. In contrast to ICR, MCR is an election-based scheme. Each node that participates in MCR (not necessarily every node in the network, as opposed to synchronous protocols) engages in a competion to become a $\mathrm{CH}$ by setting a timer called the re-clustering timer $T_{R}$. This timer is shorter if the $\mathrm{CH}$-probability is higher: at a node $i$, $T_{R}=(1-P(i)) T$, where $T$ is a system constant.

With periodic CHHELLO packet exchanges (see Sec. III-D2), each $\mathrm{CH}$ node $j$ monitors the standard deviation of node energy in its cluster and calculates the average $\overline{\sigma_{\mathcal{E}}}(j)$ of the standard deviation values collected from its $\mathrm{CH}$ neighbours. If this average is more than a certain level of the standard deviation $\sigma_{0}$ of the initial node energy levels of the network ${ }^{4}$, then $\mathrm{CH} j$ concludes that the energy balance across the region covered by neighbouring $\mathrm{CHs}$ is deteriorating. This is given by:

$$
\overline{\sigma_{\mathcal{E}}}(j)>\theta_{S T D} * \sigma_{0},
$$

where $\theta_{S T D}$ is a decision threshold coefficient. When the criterion in Eqn. 2 is met, an MCR is initiated by $\mathrm{CH} j$ which triggers its neighbouring $\mathrm{CHs}$ via a RECLUSTERCH packet to get involved. Each neighbour $\mathrm{CH}$, including $\mathrm{CH} j$ then transmits a RECLUSTER packet to their members, indicating that an MCR has started. All involved $\mathrm{CHs}$, their member nodes, as well as any other nodes in range that are not attached to a $\mathrm{CH}$, update their $\mathrm{CH}$ probability values by Eqn. 5 using the baseline probability value recorded in the packet, and then set their re-clustering timers. Upon expiry of re-clustering timers, nodes transmit ANNOUNCE packets and notify their neighbour nodes of the formation of new clusters. If a node receives an ANNOUNCE before its timer expires, it ceases to participate in the election. Successful nodes become a CH and send ACCEPT packets to those nodes from which a JOIN has been received, and confirm those nodes' cluster membership.

\section{ACP Fundamentals}

In this section, the analytical basis that enables successful cluster updates via the ICR and MCR operations is presented. First, the $\mathrm{CH}$-probability and its relation with cluster sizes and hop distances to the data sink are explained. Then, the concept of baseline probabilities is introduced as a way to balance multihop traffic loads on CHs.

1) CH-probability and cluster sizes: ACP makes a relation between a node $i$ 's $\mathrm{CH}$-probability $P(i)$ and the size of its cluster when node $i$ becomes a $\mathrm{CH}$. The cluster size of $\mathrm{CH} i$ is defined as the transmission radius denoted by $r(i)$ that $\mathrm{CH}$ $i$ uses to communicate with its cluster members. The $P(i) \Leftrightarrow$ $r(i)$ relation is a result of the fact that $\mathrm{CH}$ nodes are chosen by an election mechanism in which each node $i$ transmits an announcement packet to declare its $\mathrm{CH}$ role within a range of $r(i)$. The announcement packet effectively notifies other nodes

\footnotetext{
${ }^{4} \sigma_{0}$ is a value pre-set at each node at deployment time.
} 
that they cannot become a $\mathrm{CH}$ since they are within node $i$ 's prospective cluster boundary. Similar election mechanisms have been used in previous protocols [6] [7].

The $P(i) \Leftrightarrow r(i)$ relation is derived such that there is a high probability to have at least one other node within the range $r(i)$, given the node density conditions. In doing so, the aim is for sensors to have a high chance to locate other $\mathrm{CHs}$ in their locality. This corresponds to the average number of $\mathrm{CHs}$ in a given area, which can be modelled by the Poisson pdf using the average uniform node deployment density $\sigma$. Defining node $i$ 's announcement boundary by a circular radius $r(i)$, the probability that there is at least one $\mathrm{CH}$ node within this circular boundary is given by:

$$
\begin{aligned}
\operatorname{Prob}\{\mathbf{n} \geq 1\} & \approx 1-e^{-\sigma P(i) \pi r(i)^{2}}, \\
r(i) & \approx \sqrt{\frac{2 \ln 10}{\sigma P(i) \pi}} .
\end{aligned}
$$

where $\sigma P(i)$ is the average $\mathrm{CH}$ density in the local area, and Eqn. 4 is derived by setting Eqn. 3 to $99 \%$.

2) CH-probability and hop distances to the sink: The variables $P(i)$ and $r(i)$ are related with node $i$ 's hop distance $h(i)$ to the sink, in order to capture the effect of multihop data forwarding imbalance in a large-scale WSN. The CHs that are closer to the sink have higher data forwarding loads, which suggests that they should ideally have smaller cluster sizes so that (1) the additional load from cluster members is less, and (2) more clusters can share the high load. Therefore, ACP strives to avoid network hot-spots by adjusting the cluster size $r(i)$ according to the hop distance $h(i)$. This is possible by associating $h(i)$ with a dynamic variable, called the baseline probability, $p_{h(i)}$, which depends on the traffic loads in node $i$ 's neighbourhood. ACP relates the $\mathrm{CH}$ probability $P(i)$ to the baseline probability $p_{h(i)}$ as follows:

$$
P(i)=p_{h(i)} \frac{E(i)}{\mathcal{E}_{\max }(i)},
$$

where $\mathcal{E}_{\max }(i)$ is the maximum residual node energy among the neighbours of node $i$ (including its own energy). The energy proportion makes sure that higher energy nodes have a better chance to become a $\mathrm{CH}$.

3) Capturing the effect of multihop traffic load imbalance via 'baseline probabilities': Forwarding data flows hop by hop towards the sink causes increasing loads on the set of $\mathrm{CHs}$ at decreasing hop distances. Hence, denoting $L_{x}$ as the average per-CH load at hop $x$, we have $L_{1}<\ldots<L_{K}$. In a large-scale WSN with many nodes, approximately circular bands of regions of almost equal widths around the sink are formed [18], where each region encloses the locations where nodes at a particular hop distance to the sink are found. Denoting the largest hop distance to the sink as $K$, the total average data traffic forwarded by $\mathrm{CHs}$ at hop $x$ is $L_{x} \approx \sum_{j=i}^{K}(2 j-1) a t=\left(K^{2}-i^{2}+2 i-1\right) a t$, where $t$ is the average sensor data traffic rate, $a$ represents the area of the inner-most region, and $(2 x-1) a$ approximates the area of the region at hop $x$.
For an equal distribution of load on CHs across the network, the number of $\mathrm{CHs}$ in a hop distance region must be higher if the forwarded traffic rate by that region is higher. As such, consider two regions $R_{1}$ and $R_{2}$ with areas of $A_{1}$ and $A_{2}$, data loads of $L_{1}$ and $L_{2}$ served by $n_{1}$ and $n_{2} \mathrm{CHs}$, respectively. The corresponding $\mathrm{CH}$ probabilities are $p_{1}=\frac{n_{1}}{\sigma A_{1}}$ and $p_{2}=$ $\frac{n_{2}}{\sigma A_{2}}$. In order to have the same load per $\mathrm{CH}$ and thus have energy equalisation between the two regions, we must ensure $\frac{L_{1}}{n_{1}} \approx \frac{L_{2}}{n_{2}}$. Hence, we have $p_{2} \approx \frac{L_{2}}{L_{1}} \frac{A_{1}}{A_{2}} p_{1}$, and the baseline probability of hop distance $x$ can be represented in terms of the baseline probability of the inner-most region, as:

$$
p_{x}=\frac{L_{x}}{L_{1}} \frac{A_{1}}{A_{x}} p_{1}=\frac{K^{2}-x^{2}+2 x-1}{(2 x-1) K^{2}} p_{1} .
$$

Setting a large $p_{1}$ leads to having more $\mathrm{CHs}$ in the network. Based on Eqn. 6, the total number of $\mathrm{CHs}$ in the network is approximately $M=\sum_{j=1}^{K}(2 j-1) a p_{j} \sigma=$ $\sigma \pi R^{2} p_{1} \sum_{j=1}^{K} \frac{K^{2}-j^{2}+2 j-1}{K^{2}}$, where $R$ is the CH-to-CH transmission range. Denoting $\sum_{j=1}^{K} \frac{K^{2}-j^{2}+2 j-1}{K^{2}}=C$, we have $p_{1}=\frac{M}{C \sigma \pi R^{2}}$.

4) Network initialisation: The first step of network initialisation is the discovery of node hop distances to the data sink by a single packet INIT from the sink that is flooded in the network by all nodes with the $\mathrm{CH}$-to- $\mathrm{CH}$ transmission range $R$ [17]. Each time this packet is forwarded, an acknowledgement packet ACK is sent back by receiver nodes. Those nodes that forward INIT packets, but consistently do not receive any ACKs back, prepare a HOPNOTIFY packet and unicast it to the sink through the discovered shortest path. The sink collects all HOPNOTIFY packets, determines the largest hop distance value $K$, and estimates the baseline probability $p_{1}$ of the inner-most region. Then, it disseminates $K$ and $p_{1}$ to network nodes by flooding a SET packet.

The second step is the calculation of the initial baseline probabilities, as outlined in Sec. III-C3. Upon reception of $\mathrm{SET}$, each node $i$ computes $p_{h(i)}$ by Eqn. 6 and its initial range by $r(i)=\sqrt{\frac{2 \ln 10}{\sigma p_{h(i)} \pi}}$. After a single exhange of HELLO messages, the initial CH-probability $P(i)$ is computed by Eqn. 5. Then, the re-clustering timer (see Sec. III-B2) is set by $t_{R}(i)=(1-P(i)) T$, and nodes send ANNOUNCE packets when theirs timers expire. Finally, such nodes become $\mathrm{CHs}$, whereas receiving nodes join the elected CHs.

\section{Neighbourhood Packet Exchanges}

The operation of ACP is based on the exchange of periodic feedback packets from neighbour nodes. Besides node-specific values, such as node ID and hop distance to the sink, each periodic packet contains some information required to update energy statistics. ACP has three neighbourhood packet types: HELLO, CHHELLO, and CHSIGNAL ${ }^{5}$.

\footnotetext{
${ }^{5}$ Nodes have their own timers that control the periodic transmission of these packets and node timers are not necessarily synchronised.
} 
1) HELLO packets: Each node $i$ transmits a periodic HELLO packet with a transmission range of $r(i)$, containing the residual energy level information, $E(i)$. Node $i$ collects HELLO packets from the set of its neighbours and updates the following local statistics: (i) local energy average $\mathcal{E}(i)$, (ii) standard deviation of local residual node energy levels $\sigma_{\mathcal{E}}(i)$, and (iii) maximum local residual node energy level $\mathcal{E}_{\max }(i)$.

2) CHHELLO packets: Each $\mathrm{CH}$ node $i$ periodically transmits additional neighbourhood packets called CHHELLO to its neighbour $\mathrm{CHs}$ once in every CHHELLO time period, with the range $R$. $\mathrm{CH}$ nodes need not be synchronised. The exchange of CHHELLO packets provides co-ordination between neighbouring $\mathrm{CHs}$ and clusters, e.g. the baseline probability $p$ is updated according to changing traffic loads on $\mathrm{CHs}$ and the dynamicity of the set of CHs (see Sec. III-E1).

The CHHELLO packet contains information on: (i) hop distance to the sink $h(i)$, (ii) baseline probability $p_{h(i)}$, (iii) the most recent traffic load $L(i)$ on the $\mathrm{CH}$, and (iv) the standard deviation of local residual node energy levels $\sigma_{\mathcal{E}}(i)$. $\mathrm{CH} i$ updates the average $\overline{\sigma_{\mathcal{E}}}(i)$ of the standard deviation values collected from neighbour $\mathrm{CHs}$, which is used in re-clustering operations as an indicator of the energy variation over multiple clusters, as explained in Sec. III-B2.

3) CHSIGNAL packets: $\mathrm{CH}$ nodes periodically exchange CHSIGNAL packets to update their next hops to the sink. Each CHSIGNAL contains energy and hop distance information. These packets are also used for nodes to track the existence of $\mathrm{CHs}$ in their vicinity, hence, in contrast to CHHELLOs, they are received by not only $\mathrm{CHs}$ but also ordinary sensor nodes.

\section{E. Update of Protocol Variables}

Protocol variables are updated based on the information received from neighbourhood packets.

1) Baseline probability values: $\mathrm{CH}$ nodes refine their baseline probability values, to balance the load on $\mathrm{CHs}$, according to the latest network conditions, via the periodic CHHELLOs.

Let the set of CHs at hop distance $x$ to the sink be $S_{x}$. CHs in $S_{i}$ can receive CHHELLO packets from other CHs in $S_{i-1}$, $S_{i}$ and $S_{i+1}$, provided that there are some neighbour $\mathrm{CHs}$ in these sets. To equalise the traffic loads on individual $\mathrm{CHs}$,

$$
p_{\text {inew }} \approx \frac{L_{i}}{L_{i-1}} p_{i-1}, \quad p_{\text {inew }} \approx \frac{L_{i}}{L_{i+1}} p_{i+1},
$$

must hold, where $p_{\text {inew }}$ is the new value to be assigned to $p_{i}$. Due to dynamically changing traffic loads on $\mathrm{CHs}$, the two objectives in Eqn. 7 may be in conflict, as they may separately and simultaneously force $p_{i}$ to decrease or to increase. In order to have a unified update rule, the effects of $S_{i-1}$ and $S_{i+1}$ are scaled to be proportional to the corresponding number of neighbour CHs, namely $N_{i-1}$ and $N_{i+1}$. Hence,

$$
\begin{aligned}
p_{i} & =\sum_{k=i-1}^{i+1} \overline{p_{k}} \frac{\overline{L_{i}}}{\overline{L_{k}}}\left(\frac{N_{k}}{N_{i-1}+N_{i}+N_{i+1}}\right) \\
& =\frac{N_{i} \overline{p_{i}}+N_{i-1} \overline{p_{i-1}} \frac{\overline{L_{i}}}{\overline{L_{i-1}}}+N_{i+1} \overline{p_{i+1}} \frac{\overline{L_{i}}}{N_{i+1}}}{N_{i-1}+N_{i}+N_{i+1}},
\end{aligned}
$$

where $\overline{p_{i-1}}, \overline{p_{i}}, \overline{p_{i+1}}$ are the average baseline probability values collected by a $\mathrm{CH}$ in $S_{i}$ from its $\mathrm{CH}$ neighbours in $S_{i-1}, S_{i}, S_{i+1}$, respectively.

Each $\mathrm{CH}$ node in $S_{i}$ first extracts the information on the advertised traffic loads and the baseline probabilities from received CHHELLO packets, and also monitors $N_{i-1}, N_{i}$, and $N_{i+1}$. Then, the average load values $\overline{L_{i-1}}, \overline{L_{i}}$, and $\overline{L_{i+1}}$, as well as the averages $\overline{p_{i-1}}, \overline{p_{i}}$, and $\overline{p_{i+1}}$ are computed. Finally, the baseline probability is updated by Eqn. 8 .

2) $\mathrm{CH}$-probability and transmission range: Whenever a $\mathrm{CH}$ $i$ computes a new value for its baseline probability $p_{h(i)}, \mathrm{CH}$ $i$ also updates its $\mathrm{CH}$-probability $P(i)$ using Eqn. 5. Then, with the new value of $P(i), \mathrm{CH} i$ updates its transmission range $r(i)$ using Eqn. 4. As explained in Sec. III-E1, CH nodes periodically refresh these variables based on received CHHELLO packets. All other nodes perform these updates only during re-clustering operations.

\section{Performance Evaluation}

The performance of ACP is compared with that of a synchronous node clustering protocol, UCR [6], which is also designed for large-scale WSNs with multihop data delivery to a data sink. Similar to UCR, an ideal MAC layer and error-free communication links are assumed. In both protocols, the $\mathrm{CH}-$ to- $\mathrm{CH}$ range $R$ is calculated as in [17] to boost connectivity. Packet sizes are picked according to the carried information, as listed in Table I, where the first four rows are for ACP, the next three rows are for UCR, and the last two rows are for the common packet types.

TABLE I

ACP, UCR, AND COMMON PACKET SIZES (BYTES)

\begin{tabular}{|c|c|c|c|}
\hline Packet & Size, Content & Packet & Size, Content \\
\hline BEACON & $64,--$ & RECLUSTERCH & $64,-$ \\
\hline RECLUSTER & $96, p_{h}(i)$ & CHCHANGE & 80, New CH ID \\
\hline CHCANCEL & $64,-$ & CHSIGNAL & $112, h(i), E(i)$ \\
\hline HELLO & $96, E(i)$ & CHHELLO & $176, p_{h(i)}, L(i), \sigma \mathcal{E}(j), h(i)$ \\
\hline \hline FINALHEAD & $64,-$ & CHADV & $64,--$ \\
\hline COMPETE & $128, E(i), r(i)$ & CHBEACON & $128, E(i)$, distance to sink \\
\hline QUITELECTION & $64,-$ & & $64,--$ \\
\hline \hline ANNOUNCE & $96, r(i)$ & JOIN & \\
\hline ACCEPT & $64,-$ & & \\
\hline
\end{tabular}

\section{A. Energy Consumption Model}

The energy consumption model is adopted from prior studies [8] [10] [13], given by Eqn. 9 below, which computes the transmission energy of $l$-bits to a receiver node that is $x$ metres apart. The distance $x$ in Eqn. 9 is the transmission range that a node uses within a cluster, e.g. $x=r(i)$ from a $\mathrm{CH} i$ towards a cluster member or from $i$ 's cluster members towards $i$. For inter-cluster communications, $\mathrm{CHs}$ use a range of $x=R$.

$$
\begin{gathered}
E_{T x}=\left\{\begin{array}{cc}
l\left(e_{t}+\epsilon_{f s} x^{2}\right) J & \text { if } x \leq d_{T h} \\
l\left(e_{t}+\epsilon_{m p} x^{4}\right) J & \text { if } x>d_{T h}
\end{array}\right. \\
E_{R x}=l . e_{r}, E_{\text {Proc }}=l . E_{b i t}
\end{gathered}
$$

The model differentiates between multi-path fading and free-space conditions. Considering a distance threshold of $d_{T h}=87 m$, free-space conditions are applied with $\epsilon_{f s} x^{2}$ 
when $x \leq d_{T h}$, where $\epsilon_{f s}=10 \mathrm{pJ} / \mathrm{bit} / \mathrm{m}^{2}$. Otherwise, "multi-path" fading conditions are in place with $\epsilon_{m p} x^{4}$, where $\epsilon_{m p}=0.0013 \mathrm{pJ} / \mathrm{bit} / \mathrm{m}^{4}$. The transmission and reception energy consumption constants are $e_{t}=e_{r}=50 \mathrm{~nJ} / \mathrm{bit}$. The per-bit processing energy $E_{b i t}=5 \times 10^{-9} \mathrm{~J} /$ bit with a standard deviation of $10^{-9} \mathrm{~J} /$ bit among network nodes.

\section{B. Results}

Presented performance results are averages of 10 randomly deployed networks in an area of $400 \mathrm{~m} \times 400 \mathrm{~m}$, where the data sink is located at the centre. The initial $\mathrm{CH}$ density is $10 \%$ for both protocols. Sensors with 10 different data generation rates in [0.5 5] Kbps are considered. The initial average node energy level is $2 J$ with a standard deviation of $0.25 \mathrm{~J}$.

As in UCR, performance results are generated by MATLAB simulations. The unit-time period is the average link transmission time for a control packet of 200 bytes, corresponding to approximately $0.3 \mathrm{msec}$ for a $6 \mathrm{Mbps}$ transmission rate. Time periods are multiples of the unit-time, i.e. $\mathrm{HELLO}=40$, CHHELLO $=80$, CHSIGNAL $=50, T_{R}=1$. An ICR check is performed after a period of $T_{I N}=100$, following a successful ICR. Decision threshold coefficients for ICR and MCR are arbitrarily chosen as $\Theta_{I N}=1.25$ and $\Theta_{S T D}=1.25$.

Figure 1 illustrates the performance comparison of synchronous (UCR) and asynchronous (ACP) node clustering in multihop WSNs with respect to network lifetime, average data throughput at the sink, and node outage, for networks with various number of nodes. It is observed that an asynchronous protocol can achieve similar levels of network lifetime, while reducing the fraction of time (outage) when nodes are disconnected from the data sink and increasing the average data throughput at the sink, thanks to the exchange of neighbourhood messages and timely update of clusters at local areas.
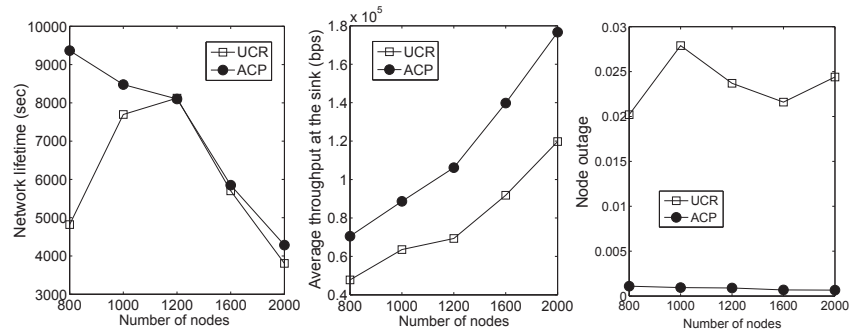

Fig. 1. Performance comparison of UCR and ACP.

\section{Conclusion}

In this paper, it is shown that when adapting the network hierarchy to dynamic traffic load conditions in a clustered multihop WSN, network-wide node synchronisation is not a necessity. Similar benefits, as achieved by synchronous clustering, can be obtained in terms of extension of network lifetime. Furthermore, making independent and local re-clustering decisions at cluster head nodes provides higher node connectivity to the network's data sink and higher data throughput at the sink, as compared to engaging all network nodes in re-clustering operations simultaneously. Hence, asynchronous node clustering is a promising technique towards achieving fully self-organised wireless sensor networks.

\section{ACKNOWLEDGMENTS}

This work is supported by the EU FP7 Project EXALTED [19] (ICT-5-258512) and the EPSRC/ChistEra CONCERT Project (EP/L018683/1).

\section{REFERENCES}

[1] A. A. Abbasi and M. Younis, "A survey of clustering algorithms for wireless sensor networks," Elsevier Computer Communications, vol. 30, no. 14-15, pp. 2826-2841, 2007.

[2] J. Baek, S. K. An, and P. Fisher, "Dynamic cluster header selection and conditional re-clustering for wireless sensor networks," IEEE Transactions on Consumer Electronics, vol. 56, no. 4, pp. 2249-2257, 2010.

[3] P. Hebden and A. R. Pearce, "Distributed asynchronous clustering for self-organisation of wireless sensor networks," in $4^{\text {th }}$ International Conference on Intelligent Sensing and Information Processing (ICISIP). IEEE, 2006, pp. 34-42.

[4] J. Luo, H.-Z. Liu, R.-F. Li, and L. Bao, "QoS-oriented asynchronous clustering protocol in wireless sensor networks," in $4^{\text {th }}$ International Conference on Wireless Communications, Networking and Mobile Computing (WiCOM). IEEE, 2008.

[5] O. Younis and S. Fahmy, "HEED: A hybrid, energy-efficient, distributed clustering approach for ad hoc sensor networks," IEEE Transactions on Mobile Computing, vol. 3, no. 9, pp. 366-379, December 2004.

[6] G. Chen, C. Li, M. Ye, and J. Wu, "An unequeal cluster-based routing protocol in wireless sensor networks," Wireless Networks, vol. 15, no. 2 , pp. 193-207, February 2009.

[7] D. Wei, Y. Jin, S. Vural, and R. Tafazolli, "An energy-efficient clustering solution for wireless sensor networks," IEEE Transactions on Wireless Communications, vol. 10, no. 11, pp. 3973-3983, November 2011.

[8] W. B. Heinzelman, A. P. Chandrakasan, and H. Balakrishnan, "An application-specific protocol architecture for wireless microsensor networks," IEEE Transactions on Wireless Communications, vol. 1, no. 4 pp. 660-670, October 2002.

[9] S. Bandyopadhyay and E. J. Coyle, "An energy efficient hierarchical clustering algorithm for wireless sensor networks," in $22^{\text {nd }}$ Annual Joint Conference of the IEEE Computer and Communications (INFOCOM), vol. 3, San Francisco, CA, USA, April 2003, pp. 1713-1723.

[10] I. Matta, G. Smaragdakis, and A. Bestavros, "SEP: A stable election protocol for clustered heterogeneous wireless sensor networks," in SANPA. Boston, MA, USA: IEEE, August 2004.

[11] V. Mhatre and C. Rosenberg, "Homogeneous vs heterogeneous clustered networks: A comparative study," in International Conference on Communications (ICC). IEEE, 2004.

[12] M. Ye, C. Li, G. Chen, and J. Wu, "EECS: An energy efficient clustering scheme in wireless sensor networks," in $24^{\text {th }}$ International Performance, Computing, and Communications Conference. Phoenix, AZ, USA: IEEE, 2005, pp. 535-540.

[13] D. Kumar, T. C. Aseri, and R. B. Patel, "EEHC: Energy efficient heterogeneous clustered scheme for wireless sensor networks," Computer Communications, vol. 32, no. 4, pp. 662-667, March 2009.

[14] L. Qing, Q. Zhu, and M. Wang, "Design of a distributed energy-efficient clustering algorithm for heterogeneous wireless sensor networks," Elsevier Computer Communications, vol. 29, pp. 2230-2237, March 2006.

[15] M. Qin and R. Zimmermann, "VCA: An energy-efficient voting-based clustering algorithm for sensor networks," Journal of Universal Computer Science, vol. 13, no. 1, pp. 87-109, January 2007.

[16] S. Soro and W. B. Heinzelman, "Prolonging the lifetime of wireless sensor networks via unequal clustering," in Proceedings of the $19^{\text {th }}$ IEEE International Parallel and Distributed Processing Symposium, vol. 13, 2005.

[17] S. Vural, P. Navaratnam, and R. Tafazolli, "Transmission range assignment for backbone connectivity in clustered wireless sensor networks," IEEE Wireless Communications Letters, pp. 46-49, February 2013.

[18] S. Nath, V. Ekambaram, A. Kumar, and P. V. Kumar, "Theory and algorithms for hop-count-based localization with random geometric graph models for dense sensor networks," ACM Transactions on Sensor Networks, no. 8, pp. 1-38, 2012.

[19] EU-FP7, "Expanding LTE for devices," http://www.ict-exalted.eu/, 2010-2013. 